\title{
The parameter distributions of the integer GPS model
}

\author{
P. J. G. Teunissen \\ Department of Mathematical Geodesy and Positioning, Delft University of Technology, \\ Thijsseweg 11, 2629 JA Delft, The Netherlands \\ e-mail: p.j.g. teunissen@citg.tudelft.nl; Tel.: +31-15-278-2558; Fax: +31-15-278-3711
}

Received: 30 January 2001 / Accepted: 31 July 2001

\begin{abstract}
A parameter estimation theory is incomplete if no rigorous measures are available for describing the uncertainty of the parameter estimators. Since the classical theory of linear estimation does not apply to the integer GPS model, rigorous probabilistic statements cannot be made with reference to the classical results. The fact that integer parameters are involved in the estimation process forces a reappraisal of the propagation of uncertainty. It is with this purpose in mind that the joint and marginal distributional properties of both the integer and non-integer parameters of the GPS model are determined. These joint distributions can also be used to determine the distribution of functions of the parameters. As an important example, the distribution of the vector of ambiguity residuals is determined.
\end{abstract}

Key words: GPS - Ambiguity Resolution Integer/non-integer Parameter Distributions

\section{Introduction}

Any GPS model of observation equations which makes use of carrier phase data and which is based on the use of two or more receivers, can be parameterized in integers and non-integers. The integer parameters refer to the unknown cycle ambiguities of the doubledifference (DD) carrier phase data, while the non-integer parameters refer to the baseline components and possibly additional parameters such as atmospheric delays. When the integerness of the integer parameters is explicitly taken into account in the parameter estimation process, we speak of carrier phase ambiguity resolution. Carrier phase ambiguity resolution applies to a great variety of GPS models which are currently in use in navigation, surveying, geodesy and geophysics. An overview of these models, together with their applica- tions, can be found in textbooks such as Leick (1995), Parkinson and Spilker (1996), Hofmann-Wellenhof et al. (1997), Strang and Borre (1997) and Teunissen and Kleusberg (1998).

As with any parameter estimation process, it is not enough to simply estimate the parameters and be done with it. We also need to have a way of inferring the uncertainty of the parameter solution. In the classical theory of linear estimation this is most often done by means of variance matrices. For the classical theory this makes sense. After all, when the model is linear and when the data are normally (Gaussian) distributed, the linear parameter estimators will be normally distributed as well. And since the peakedness of a multivariate normal distribution is completely captured by its variance matrix, it suffices to use variance matrices as a precision description of the parameters. This relatively simple approach fails to hold, however, in the case of integer GPS model. The fact that integer parameters are involved in the estimation process will result in non-Gaussian distributions even when the model is linear and the data are normally distributed. Hence, in order to obtain a rigorous description of the parameter uncertainty, we will have to bypass the use of variance matrices and go directly to the parameter distributions themselves. It is the purpose of this contribution to determine these parameter distributions.

In this contribution we use the term 'distribution' in the usual generic sense. For concrete cases, however, we describe the way in which the distribution of the random variate is specified. This is often in the form of a probability density function (PDF) or in the form of a probability mass function (PMF). The contribution is organized as follows. In Sect. 2 we formulate the integer GPS model and give a brief review of the principles involved in ambiguity resolution. The steps of integer estimation together with the class of admissible integer estimators are described. In Sect. 3 we determine the various parameter distributions. They hold true for any choice of integer estimator from the class of admissible ambiguity estimators. A visualization of the various 
parameter distributions is also given. We first formulate our starting assumptions and then determine the joint distribution of the 'fixed' and 'float' ambiguities. This joint distribution can then be used to determine the corresponding marginal and conditional distributions. We also use it to determine the distribution of the vector of ambiguity residuals. This distribution is needed in case we want to test for the integerness of the ambiguities. Following the joint distribution of the 'fixed' and 'float' ambiguities, we determine the joint distributions of these ambiguities and the 'float' and 'fixed' baseline estimators. These distributions allow us to determine the probabilistic dependence between the baseline solution and the ambiguities. The joint distribution is then used to determine the marginal distribution of the 'fixed' baseline estimator. This distribution captures the complete probabilistic characteristics of the 'fixed' baseline. It is finally shown how this distribution can be used to construct unconditional confidence regions for the 'fixed' baseline.

\section{The integer GPS model}

Any GPS model can be cast in the following system of linear(ized) observation equations:

$y=A a+B b+e$

where $y$ is the given GPS data vector of order $m, a$ and $b$ are the unknown parameter vectors respectively of order $n$ and $p$, and $e$ is the noise vector. The data vector $y$ will usually consist of the 'observed minus computed' single-, dual- or triple-frequency DD phase and/or pseudorange (code) observations accumulated over all observation epochs. The entries of vector $a$ are then the DD carrier phase ambiguities, expressed in units of cycles rather than range. They are known to be integers, $a \in Z^{n}$. The entries of the vector $b$ will consist of the remaining unknown parameters, such as for instance baseline components (coordinates) and possibly atmospheric delay parameters (troposphere, ionosphere). They are known to be real-valued, $b \in R^{p}$. Although vector $b$ may contain other or more real-valued unknown parameters than only those of the baseline(s), we will in this contribution, as a matter of terminology, still call its estimator the baseline estimator.

The procedure which is usually followed for solving the GPS model of Eq. (1) can be divided into three steps (Teunissen 1993). In the first step we simply discard the integer constraints $a \in Z^{n}$ on the ambiguities and perform a standard adjustment. As a result we obtain the (real-valued) estimates of $a$ and $b$, together with their variance-covariance matrix

$$
\left[\begin{array}{l}
\hat{a} \\
\hat{b}
\end{array}\right], \quad\left[\begin{array}{cc}
Q_{\hat{a}} & Q_{\hat{a} \hat{b}} \\
Q_{\hat{b} \hat{a}} & Q_{\hat{b}}
\end{array}\right]
$$

This solution is referred to as the 'float' solution. In the second step the 'float' ambiguity estimate $\hat{a}$ is used to compute the corresponding integer ambiguity estimate

$\check{a}=S(\hat{a})$ with $S: R^{n} \mapsto Z^{n}$ a mapping from the $n$-dimensional space of real numbers to the $n$-dimensional space of integers. Once the integer ambiguities are computed, they are used in the third and final step to correct the 'float' estimate of $b$. As a result we obtain the ambiguity resolved baseline solution

$\check{b}=\hat{b}-Q_{\hat{b} \hat{a}} Q_{\hat{a}}^{-1}(\hat{a}-\check{a})$

This solution is usually referred to as the 'fixed' baseline. Both Eq. (3) and Eq. (4) depend on the choice of integer estimator. Different choices of the map $S: R^{n} \mapsto Z^{n}$ will result in different integer estimators and will thus also produce differences in the probability distribution of the estimators.

There exists a whole class of integer estimators from which we can choose. In order to introduce this class, we start from the map $S: R^{n} \mapsto Z^{n}$. Due to the discrete nature of $Z^{n}$, the map $S$ will not be one-to-one, but instead a many-to-one map. This implies that different realvalued ambiguity vectors will be mapped to the same integer vector. We can therefore assign a subset $S_{z} \subset R^{n}$ to each integer vector $z \in Z^{n}$

$S_{z}=\left\{x \in R^{n} \mid z=S(x)\right\}, \quad z \in Z^{n}$

The subset $S_{z}$ contains all real-valued ambiguity vectors that will be mapped by $S$ to the same integer vector $z \in Z^{n}$. This subset is referred to as the pull-in region of $z$ (Jonkman 1998; Teunissen 1998a). It is the region in which all ambiguity 'float' solutions are pulled to the same 'fixed' ambiguity vector $z$. Thus $\check{a}=z \Longleftrightarrow \hat{a} \in S_{z}$. By using the indicator function of the pull-in regions, the integer ambiguity estimator can be expressed as

$\check{a}=\sum_{z \in Z^{n}} z s_{z}(\hat{a}) \quad$ with $s_{z}(x)=\left\{\begin{array}{cc}1 & \text { if } x \in S_{z} \\ 0 & \text { otherwise }\end{array}\right.$

Since the pull-in regions define the integer estimator completely, we can define classes of integer estimators by imposing various conditions on the pull-in regions. The class of admissible integer ambiguity estimators is defined as follows.

Definition (Admissible integer estimators). The integer estimator $\check{a}=\sum_{z \in Z^{n}} z s_{z}(\hat{a})$ is said to be admissible when its pull-in regions $S_{z}=\left\{x \in R^{n} \mid z=S(x)\right\}, z \in Z^{n}$, satisfy

(i) $\cup_{z \in Z^{n}} S_{z}=R^{n}$

(ii) Int $S_{z_{1}} \cap \operatorname{Int} S_{z_{2}}=\emptyset, \forall z_{1}, z_{2} \in Z^{n}, z_{1} \neq z_{2}$

(iii) $S_{z}=z+S_{0}, \forall z \in Z^{n}$

where 'Int' denotes the interior of the subset. For the motivation of the above definition we refer to Teunissen (1999). Examples of integer estimators that belong to the above class are integer rounding, integer bootstrapping and integer least-squares (LS). For the material that follows, it is useful to note that the indicator functions of admissible pull-in regions fulfil the two basic conditions of PMFs and PDFs, namely of being non-negative and having an area of 1 . For fixed $x \in R^{n}, s_{z}(x)$ fulfils the PMF conditions 
(i) $s_{z}(x) \geq 0, \forall z \in Z^{n}$

(ii) $\sum_{z \in Z^{n}} s_{z}(x)=1$

and for fixed $z \in Z^{n}, s_{z}(x)$ also fulfils the PDF conditions

(i) $s_{z}(x) \geq 0, \forall x \in R^{n}$

(ii) $\int_{R^{n}} s_{z}(x) \mathrm{d} x=1$

The last property follows from the fact that the area or volume of admissible pull-in regions equals 1 . This can be seen as follows. Let $C_{0} \subset R^{n}$ be the origin-centred unit cube and let $S_{z} \subset R^{n}$ be an admissible pull-in region. Since the volume of the unit cube equals 1 and admissible pull-in regions cover $R^{n}$ without gaps and overlaps, we have $1=\sum_{z \in Z^{n}} \int_{S_{z} \cap C_{0}} \mathrm{~d} x$. Application of the change of variable $x=y+z$ gives $1=\sum_{z \in Z^{n}} \int_{S_{0} \cap C_{-z}} \mathrm{~d} y$. From this it follows that $1=\int_{S_{0}} \mathrm{~d} y$, since $R^{n^{0}}$ is also covered without gaps and overlaps by the translated copies of the unit cube. We therefore have $\int_{R^{n}} s_{z}(x) \mathrm{d} x=\int_{S_{z}} \mathrm{~d} x=\int_{S_{0}} \mathrm{~d} x=1$.

\section{The parameter distributions}

In this section we will determine the parameter distributions of the integer GPS model. In order to do so, we first need to specify our probabilistic assumptions about the 'float' solution. In this contribution we will assume that the 'float' solutions are normally (Gaussian) distributed as

$$
\left[\begin{array}{l}
\hat{a} \\
\hat{b}
\end{array}\right] \sim \mathrm{N}\left(\left[\begin{array}{l}
a \\
b
\end{array}\right],\left[\begin{array}{cc}
Q_{\hat{a}} & Q_{\hat{a} \hat{b}} \\
Q_{\hat{b} \hat{a}} & Q_{\hat{b}}
\end{array}\right]\right)
$$

This implies that the joint PDF of the 'float' solution is given as

$f_{\hat{b}, \hat{a}}(y, x)=f_{\hat{b} \mid \hat{a}}(y \mid x) f_{\hat{a}}(x)$

in which the marginal and conditional PDF are given as

$$
\begin{aligned}
& f_{\hat{a}}(x)=\frac{1}{\sqrt{\operatorname{det} Q_{\hat{a}}}(2 \pi)^{\frac{1}{2} n}} \exp \left\{-\frac{1}{2}\|x-a\|_{Q_{\hat{a}}}^{2}\right\} \\
& f_{\hat{b} \mid \hat{a}}(y \mid x)=\frac{1}{\sqrt{\operatorname{det} Q_{\hat{b} \mid \hat{a}}}(2 \pi)^{\frac{1}{2} p}} \exp \left\{-\frac{1}{2}\left\|y-b_{x}\right\|_{Q_{\hat{b} \mid \hat{a}}}^{2}\right\}
\end{aligned}
$$

with conditional mean $b_{x}=b+Q_{\hat{b} \hat{a}} Q_{\hat{a}}^{-1}(x-a)$ and conditional variance matrix $Q_{\hat{b} \mid \hat{a}}=Q_{\hat{b}}-Q_{\hat{b} \hat{a}} Q_{\hat{a}}^{-1} Q_{\hat{a} \hat{b}}$. Above we have used the following notation for the weighted squared norm: $\|\cdot\|_{M}^{2}=(\cdot)^{T} M^{-1}(\cdot)$, with matrix $M$ being positive definite.

\subsection{The ambiguities}

We are now ready to determine the joint distribution of the 'float' ambiguities, $\hat{a}$, and the 'fixed' ambiguities, $\check{a}$. This joint distribution can then be used to determine the corresponding marginal and conditional distributions. It can also be used to determine the distribution of functions of the 'float' and 'fixed' ambiguities. As one such application we will determine the distribution of the vector of ambiguity residuals. The joint distribution of $\hat{a}$ and $\check{a}$ will be denoted as $f_{\hat{a}, \check{a}}(x, z)$.

Theorem 1. Let $f_{\hat{a}}(x)$ be the PDF of $\hat{a}$ and let the integer ambiguity estimator be defined as $\check{a}=\sum_{z \in Z^{n}} z s_{z}(\hat{a})$, with $S_{z}(x)$ the indicator function of the pull-in region $S_{z} \subset R^{n}$, $z \in Z^{n}$. The joint distribution of $\hat{a}$ and $\check{a}$ is then given as

$f_{\hat{a}, \breve{a}}(x, z)=f_{\hat{a}}(x) s_{z}(x), \quad x \in R^{n}, \quad z \in Z^{n}$

Proof: see Appendix.

The joint distribution $f_{\hat{a}, \check{a}}(x, z)$ captures the complete probabilistic properties of the 'float' and 'fixed' ambiguities. From it the marginal distributions of $\hat{a}$ and $\check{a}$ can be recovered by integrating or summing over respectively $x$ and $z$. Hence, the PDF of $\hat{a}$ can be recovered as $\sum_{z \in Z^{n}} f_{\hat{a}, \breve{a}}(x, z)=f_{\hat{a}}(x)$ since $\sum_{z \in Z^{n}} s_{z}(x)=1$ for all $x \in R^{n}$. The distribution of the integer ambiguity estimator $\check{a}$ will not be a PDF, but a PMF. It follows as

$\int_{R^{n}} f_{\hat{a}, \check{a}}(x, z) \mathrm{d} x=\int_{S_{z}} f_{\hat{a}}(x) \mathrm{d} x=P[\check{a}=z]$

The probability $P[\check{a}=z], z \in Z^{n}$, equals the integral of the PDF of the ambiguity 'float' solution over the pull-in region $S_{z} \subset R^{n}$. The probability of correct integer estimation, also referred to as the ambiguity success rate, is given as $P[\check{a}=a]$.

In order to visualize the relationship between the three distributions $f_{\hat{a}}(x), f_{\hat{a}, \breve{a}}(x, z)$ and $P[\check{a}=z]$, we have plotted all three of them in Fig. 1 for the one-dimensional $(1-\mathrm{D})$ case $(n=1)$. The $\mathrm{PDF} f_{\hat{a}}(x)$ is plotted along the $x$-axis (left-hand plot), the PMF $P[\check{a}=z]$ is plotted
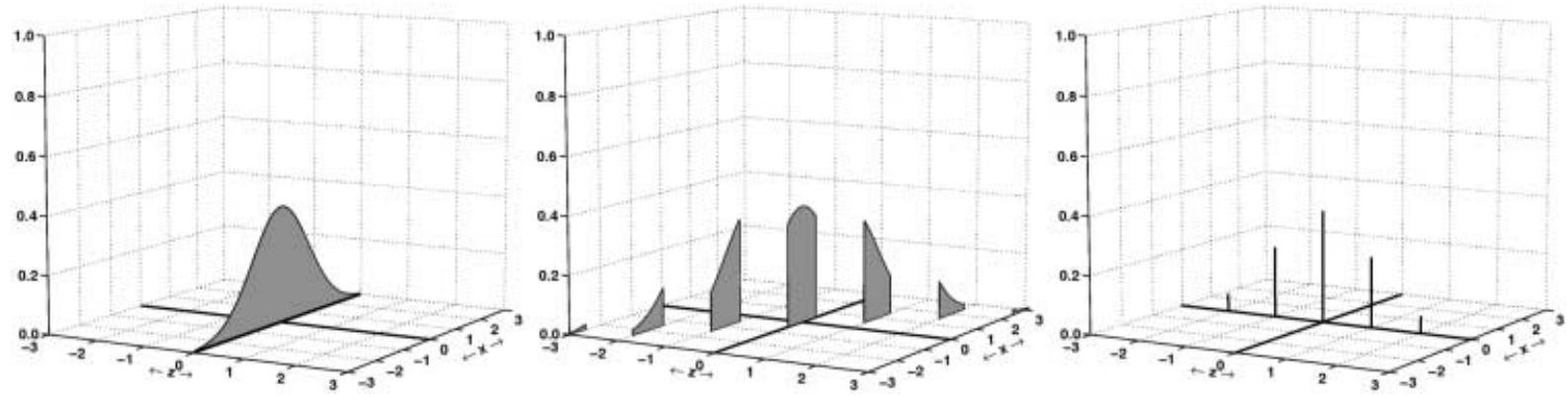

Fig. 1. The joint and marginal distributions of $\hat{a}$ and $\check{a}$ : $\operatorname{PDF} f_{\hat{a}}(x)$ (left); PDF $f_{\hat{a} \check{a}}(x, z)$ (centre); and PMF $P[\check{a}=z]$ (right) 
along the $z$-axis (right-hand plot) and the joint distribution $f_{\hat{a}, \breve{a}}(x, z)$ is plotted on the $x z$-plane (centre plot). This figure clearly shows how the joint distribution can be constructed once the pull-in regions and the PDF of the 'float' solution are given. In the 1-D case the pull-in regions take a very simple shape. In that case they are given as the integer-centred intervals of length 1 : $S_{z}=\left\{x \in R|| x-z \mid \leq \frac{1}{2}\right\}$. For each integer, say $w \in Z^{n}$, the contribution to the joint distribution equals $f_{\hat{a}}(x) s_{w}(x)$. It is obtained by first slicing out the part of the 'float' distribution which is located at the pull-in region $S_{w}$, followed by translating this slice parallel to the $z$-axis to the position $(w, w)$. The joint distribution is thus continuous along the $x$-axis, but discrete along the $z$-axis. The marginal distribution $f_{\hat{a}}(x)$ can then be recovered from the joint distribution by summing over $z$, while the PMF of $\check{a}$ is formed by integrating the joint distribution over $x$ (see Fig. 1c).

From the joint distribution we can also easily determine the conditional distributions. As an illustration we will consider the conditional distribution $f_{\tilde{a} \mid \hat{a}}(z \mid x)$. The conditional distribution of the integer ambiguities, given the 'float' estimate $\hat{a}=x$, follows from dividing the joint distribution by $f_{\hat{a}}(x)$

$f_{\tilde{a} \mid \hat{a}}(z \mid x)=s_{z}(x), \quad x \in R^{n}, z \in Z^{n}$

It equals the indicator function of the pull-in regions. It therefore implicitly defines the choice of integer estimator. The conditional distribution $f_{\tilde{a} \mid \hat{a}}(z \mid x)$ equals 1 when $x \in S_{z}$ and is zero otherwise. The conditional mean of the integer ambiguities is given as $E\{\check{a} \mid \hat{a}=x\}$ $=\sum_{z \in Z^{n}} z f_{\breve{a} \mid \hat{a}}(z \mid x)=\sum_{z \in Z^{n}} x s_{z}(x)$. From a comparism of this result with the definition of the integer ambiguity estimator of Eq. (6), it follows that $\check{a}=E\{\check{a} \mid \hat{a}\}$. The integer ambiguity estimator $\breve{a}$ is thus equal to the conditional mean in the case that the latter is interpreted as a function of the random vector $\hat{a}$.

\subsection{The ambiguity residual}

The joint distribution of Eq. (10) can also be used to determine the distribution of functions of $\hat{a}$ and $\check{a}$. An important example of such a function is the ambiguity residual. We define it as

$\check{\epsilon}=\hat{a}-\check{a}$

We will determine the distribution of $\check{\epsilon}$ in two steps. We first determine the joint distribution of $\check{\epsilon}$ and $\check{a}$ and then determine the marginal distribution $f_{\tilde{\epsilon}}(x)$ by means of summation. In deriving the joint distribution, we make use of the following transformation law for probability density functions. Let two random vectors $u$ and $v$ be related as $v=T u+t$, with $T$ and $t$ known, and matrix $T$ invertible. The PDF of $v$ can then be expressed in the PDF of $u$ as $f_{v}(v)=\left|\operatorname{det} T^{-1}\right| f_{u}\left(T^{-1}(v-t)\right)$.

By using the invertible transformation

$\left[\begin{array}{c}\check{\epsilon} \\ \check{a}\end{array}\right]=\left[\begin{array}{cc}I_{n} & -I_{n} \\ 0 & I_{n}\end{array}\right]\left[\begin{array}{l}\hat{a} \\ \check{a}\end{array}\right]$ we can express the joint distribution of $\check{\epsilon}$ and $\check{a}$ in terms of the joint distribution of $\hat{a}$ and $\check{a}$. This gives $f_{\breve{\epsilon}, \breve{a}}(x, z)=f_{\hat{a}, \breve{a}}(x+z, z)$. If we now make use of Eq. (10), we obtain $f_{\breve{\epsilon}, \breve{a}}(x, z)=f_{\hat{a}}(x+z) s_{0}(x), \quad x \in R^{n}$, $z \in Z^{n}$. The PDF of the ambiguity residuals then follows from summing this joint distribution over all integers

$f_{\check{\epsilon}}(x)=\sum_{z \in Z^{n}} f_{\hat{a}}(x+z) s_{0}(x), \quad x \in R^{n}, \quad z \in Z^{n}$

In order to show how the distribution of the ambiguity residuals is constructed from the distribution of the 'float' ambiguities, a visualization of the steps involved is given in Fig. 2 for the 1-D case $(n=1)$. Figure 2 shows the four distributions, $f_{\hat{a}}(x)$ (top left), $f_{\hat{a}, \breve{a}}(x, z)$ (top right), $f_{\tilde{\epsilon}, \breve{a}}(x, z)$ (bottom left) and $f_{\check{\epsilon}}(x)$ (bottom right). For each integer $z \in Z^{n}$ the joint distribution $f_{\hat{a}, \breve{a}}(x, z)$ is composed of slices from the marginal distribution $f_{\hat{a}}(x)$ located at $(z, z)$. Translating these slices parallel to the $x$-axis to the line $x=0$ gives the joint PDF $f_{\breve{\epsilon}, \breve{a}}(x, z)$. A further translation along the $z$-axis to the origin then finally provides $f_{\tilde{\epsilon}}(x)$.

The distribution of the ambiguity residuals is clearly non-Gaussian. We have $f_{\tilde{\epsilon}}(x)=0$ for all $x \notin S_{0}$. This implies that the norm of the vector of ambiguity residuals is always bounded irrespective of the values taken by the ambiguity 'float' solution $\hat{a}$. For the 1-D case we have $\check{\epsilon} \in[-1 / 2,+1 / 2]$. For the higher-dimensional case the bound depends on the shape of the pull-in region and therefore on the type of integer estimator chosen. The fact that the ambiguity residuals are bounded has an important implication. It implies that the difference between the 'float' baseline solution $\hat{b}$ and the 'fixed' baseline solution $\check{b}$ is also bounded, irrespective of the values taken by the 'float' ambiguities.

The distribution $f_{\tilde{\epsilon}}(x)$ can have different shapes. There are two extreme cases between which we can discriminate, namely the uniform distribution and the impulse function distribution. In order to understand these two extreme cases, consider what happens when the precision of the 'float' ambiguities, and thereby the peakedness of $f_{\hat{a}}(x)$, is varied. When the 'float' distribution $f_{\hat{a}}(x)$ becomes more peaked, which happens when the 'float' ambiguities became more precise, the PDF $f_{\check{\epsilon}}(x)$ becomes more peaked as well. However, since all the probability mass of $f_{\bar{\epsilon}}(x)$ is located within the pull-in region $S_{0}$, the peakedness of $f_{\tilde{\epsilon}}(x)$ will only start to manifest itself when $f_{\hat{a}}(x)$ is sufficiently peaked in relation to the size of the pull-in region. When this is not the case, the distribution of the ambiguity residuals will remain flat and therefore be close to $s_{0}(x)$, which is the uniform distribution for the pull-in region $S_{0}$. The 'float' distribution $f_{\hat{a}}(x)$ may be considered peaked in relation to the size of the pull-in region, when most of its probability mass is located within $S_{0}$. This happens when the ambiguity success rate is sufficiently close to 1 , in which case the two distributions will also not differ by much. Further improvement of the precision of the ambiguities will then in the limit produce an impulse function for both $f_{\hat{a}}(x)$ and $f_{\hat{\epsilon}}(x)$.

The distribution of the ambiguity residuals is symmetric and independent of the unknown integer ambi- 

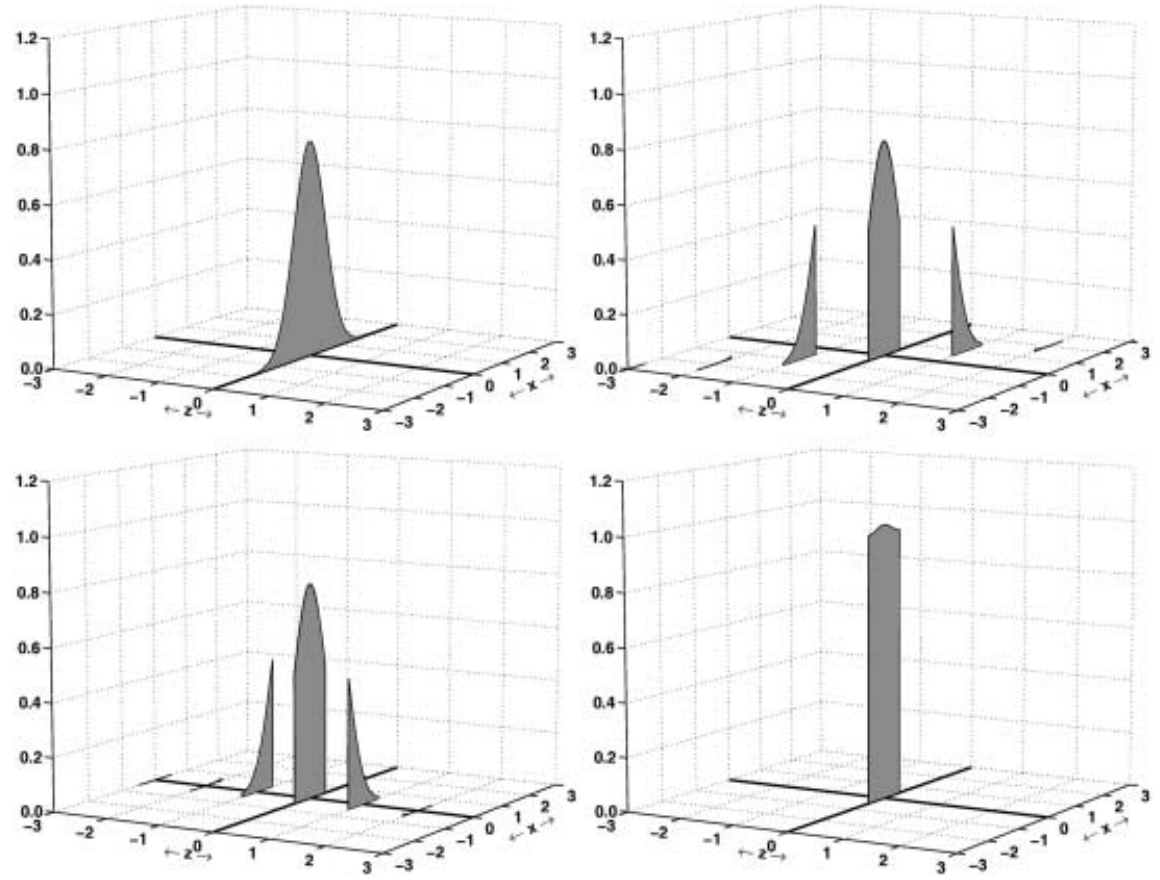

Fig. 2. The construction of $f_{\tilde{\epsilon}}(x)$ from $f_{\hat{a}}(x)$ : $\operatorname{PDF} f_{\hat{a}}(x)$ (top left); joint PDF $f_{\hat{a}, \tilde{a}}(x, z)$ (top right); joint PDF $f_{\check{\epsilon} \check{a}}(x, z)$ (bottom left); and $\operatorname{PDF} f_{\tilde{\epsilon}}(x)$ (bottom right)

guity vector $a \in Z^{n}$. Its symmetry is inherited from the 'float' distribution $f_{\hat{a}}(x)$, while the independence of $a \in Z^{n}$ follows from $\sum_{z \in Z^{n}} f_{\hat{a}}(x+a+z)=\sum_{z \in Z^{n}} f_{\hat{a}}$ $(x+z)$. The point of symmetry of the distribution is the origin. This implies that the mean of the ambiguity residual equals zero

$E\{\check{\epsilon}\}=0$

This result, combined with the fact the PDF of $\check{\epsilon}$ is completely known once the precision of the 'float' ambiguities is given, and once the choice of integer ambiguity estimator is made, allows us for the first time to formulate rigorous tests for the integerness of the parameters.

\subsection{The ambiguities and the baseline}

In order to understand the probabilistic dependence of the 'float' and 'fixed' baseline estimators on the 'float' and 'fixed' ambiguities, we will now determine the joint distributions $f_{\hat{b}, \hat{a}, \breve{a}}(u, v, w)$ and $f_{\breve{b}, \hat{a}, \breve{a}}(u, v, w)$.

Theorem 2. Let $f_{\hat{a}, \breve{a}}(v, w)=f_{\hat{a}}(v) s_{w}(v)$ be the joint distribution of $(\hat{a}, \check{a})$ and let $f_{\hat{b} \mid \hat{a}}(u \mid v)$ be the conditional distribution of $\hat{b}$ given $\hat{a}=v$. Then

$f_{\hat{b}, \hat{a}, \breve{a}}(u, v, w)=f_{\hat{b} \mid \hat{a}}(u \mid v) f_{\hat{a}, \breve{a}}(v, w)$

$f_{\breve{b}, \hat{a}, \breve{a}}(u, v, w)=f_{\hat{b} \mid \hat{a}}(u \mid w) f_{\hat{a}, \tilde{a}}(v, w)$

with $u \in R^{p}, v \in R^{n}$ and $w \in Z^{n}$.

Proof: see Appendix.

Note that the two expressions of Eq. (16) look very similar. The two distributions are both equal to zero when $v \notin S_{w}$. This follows since the indicator function $s_{w}(v)$ is zero when $v \notin S_{w}$. The two distributions are also equal when $v=w$. This corresponds with the situation that the 'float' ambiguities will be integers. In all other cases, however, the two distributions will differ. For $v \in S_{w}$ we have

$f_{\hat{b}, \hat{a}, \check{a}}(u, v, w)=f_{\hat{b} \mid \hat{a}}(u \mid v) f_{\hat{a}}(v)$

$f_{\breve{b}, \hat{a}, \breve{a}}(u, v, w)=f_{\hat{b} \mid \hat{a}}(u \mid w) f_{\hat{a}}(v)$

This shows that $f_{\hat{b}, \hat{a}, \tilde{a}}(u, v, w)$ is equal to the joint distribution $f_{\hat{b}, \hat{a}}(u, v)$ when $v \in S_{w}$, but that $f_{\breve{b}, \hat{a}, \tilde{a}}(u, v, w)$ is not.

Using Eq. (16) we are now in the position to determine the various types of joint distributions of ambiguities and baseline. They read

$$
\begin{aligned}
& f_{\hat{b}, \hat{a}}(u, v)=f_{\hat{b} \mid \hat{a}}(u \mid v) f_{\hat{a}}(v) \\
& f_{\breve{b}, \hat{a}}(u, v)=f_{\hat{b} \mid \hat{a}}(u \mid w) f_{\hat{a}}(v) \quad \text { when } v \in S_{w} \\
& f_{\hat{b}, \tilde{a}}(u, w)=\int_{S_{w}} f_{\hat{b} \mid \hat{a}}(u \mid v) f_{\hat{a}}(v) \mathrm{d} v \\
& f_{\breve{b}, \tilde{a}}(u, w)=f_{\hat{b} \mid \hat{a}}(u \mid w) \int_{S_{w}} f_{\hat{a}}(v) \mathrm{d} v
\end{aligned}
$$

The first expression of Eqs. (17) follows from using the definition of a conditional density, the second expression follows from summing $f_{\breve{b}, \hat{a}, \breve{a}}(u, v, w)$ over $w$, while the last two expressions follow from integrating $f_{\hat{b}, \hat{a}, \tilde{a}}(u, v, w)$ and $f_{\breve{b}, \hat{a} . \breve{a}}(u, v, w)$ respectively over $v$. Note that the first two distributions of Eqs. (17) are continuous along both axes, whereas the last two distributions are continuous along the $u$-axis, but discrete along the $w$-axis.

Although all of the above four distributions depend on both $f_{\hat{b} \mid \hat{a}}(u \mid v)$ and $f_{\hat{a}}(v)$, they differ in the way the probability masses are propagated. For instance, when we compare the joint distribution of the 'float' solution, $f_{\hat{b}, \hat{a}}(u, v)$, with the joint distribution of the 'fixed' solu- 
tion, $f_{\breve{b} . \breve{a}}(u, w)$, we observe in case of the latter that all the probability masses of $f_{\hat{a}}(v)$ located above the pull-in region $S_{w}$ are assigned to the integer vector $w$.

From Eqs. (17) we may also determine the various type of conditional baseline distributions. We can then infer how these distributions differ from the conditional distribution $f_{\hat{b} \mid \hat{a}}(u \mid v)$. We have

$$
\begin{array}{ll}
f_{\breve{b} \mid \hat{a}}(u \mid v)=f_{\hat{b} \mid \hat{a}}(u \mid w) & \text { when } v \in S_{w} \\
f_{\hat{b} \mid \check{a}}(u \mid w)=f_{\hat{b} \mid \hat{a}}(u \mid w) & \text { when } f_{\hat{a}}(v)=\delta(v-w) \\
f_{\breve{b} \mid \check{a}}(u \mid w)=f_{\hat{b} \mid \hat{a}}(u \mid v) & \text { when } v=w \in Z^{n}
\end{array}
$$

The first of Eqs. (18) reveals an interesting robustness property of the 'fixed' baseline. It shows that the conditional distribution of the 'fixed' baseline is insensitive to changes in the values of the ambiguity 'float' solution as long as these 'float' solutions remain within the same pull-in region. This property has important implications for the robustness of the 'fixed' baseline against biases in the ambiguity 'float' solution. These implications have been studied in Teunissen (2001). Practical results on the bias robustness of GPS ambiguity resolution have been reported in Teunissen et al. (2000).

The second equation shows that a conditioning of the 'float' baseline on $\check{a}=w$ is identical to a conditioning on $\hat{a}=w$ provided the PDF $f_{\hat{a}}(v)$ equals the impulse function centred at $w$. This result makes sense, because $f_{\hat{a}}(v)=\delta(v-w)$ implies that all the probability mass of the 'float' solution is centred at $w$.

\subsection{The baseline}

Using the above results, we are now in a position to make a start on the probabilistic evaluation of the quality of the 'fixed' baseline estimator. The third of Eqs. (18) shows that a conditioning of the 'fixed' baseline on the integer estimator $\check{a}$ is identical to a conditioning of the 'float' baseline on the 'float' ambiguity estimator $\hat{a}$, provided the conditioning is done on the same integer vector $w$. This result can be used for computing conditional confidence regions for the 'fixed' baseline solution. With $E \subset R^{p}$, we obtain from using the last of Eqs. (18) that

$$
\begin{aligned}
P[\check{b} \in E \mid \check{a}=w] & =\int_{E} f_{\breve{b} \mid \check{a}}(u \mid w) \mathrm{d} u \\
& =\int_{E} f_{\hat{b} \mid \hat{a}}(u \mid w) \mathrm{d} u, \quad w \in Z^{n}
\end{aligned}
$$

Since the conditional PDF $f_{\hat{b} \mid \hat{a}}(u \mid w)$ equals the distribution $\mathrm{N}\left[b+Q_{\hat{b} \hat{a}} Q_{\hat{a}}^{-1}(w-a), Q_{\hat{b} \mid \hat{a}}\right]$, it follows that when $E$ is chosen as the $b$-centred ellipsoid $E_{b}^{\beta}=\left\{u \in R^{p} \mid \|\right.$ $\left.u-b \|_{Q_{\hat{b} \mid \hat{a}}}^{2} \leq \beta^{2}\right\}$

$$
\begin{aligned}
& P\left[\check{b} \in E_{b}^{\beta} \mid \check{a}=w\right]=P\left[\chi_{p, \lambda_{w}}^{2} \leq \beta^{2}\right] \text { with } \\
& \lambda_{w}=\left\|Q_{\hat{b} \hat{a}} Q_{\hat{a}}^{-1}(w-a)\right\|_{Q_{b \mid \hat{a}}}^{2}, \quad w \in Z^{n}
\end{aligned}
$$

in which $\chi_{p, \lambda_{w}}^{2}$ denotes the noncentral $\chi^{2}$ distribution with $p$ degrees of freedom and noncentrality parameter $\lambda_{w}$.

The result of Eq. (20) can be used to study the probabilistic sensitivity of the conditional 'fixed' baseline for different choices of $w \in Z^{n}$. The ellipsoid $E_{b}^{\beta}$ is centred at the true but unknown baseline $b$ and its size is governed by $\beta$. Hence by choosing $\beta$ and $w \in Z^{n}$, we can compute by means of Eq. (20) the conditional probability that the 'fixed' baseline solution will still reside in $E_{b}^{\beta}$. Since the tail of a noncentral $\chi^{2}$ distribution becomes thicker for larger values of the noncentrality parameter, the conditional probability of Eq. (20) becomes smaller when $\lambda_{w}$ gets larger.

We can also follow the reverse route. By choosing $w \in Z^{n}$ and by setting the conditional probability of Eq. (20) at a certain reference value, we can compute the corresponding value for $\beta$ and thereby the size of the corresponding conditional confidence region. This size will increase for increasing values of the noncentrality parameter. It will therefore increase the more the chosen integer vector $w$ differs from the true, but unknown, integer ambiguity vector $a \in Z^{n}$.

Since Eq. (20) is a conditional probability, it does not give a complete description of the quality of the 'fixed' baseline. It only describes the quality of the 'fixed' baseline under the assumption that the integer ambiguities are known. Hence, it does not take the uncertainty of the integer ambiguity estimator into account. In order to obtain a complete description, we need instead of the conditional distribution the marginal distribution of the 'fixed' baseline, $f_{\breve{b}}(u)$. Since $f_{\breve{b}}(u)=\sum_{w \in Z^{n}} f_{\breve{b}, \breve{a}}(u, w)$, the marginal distribution follows from the last expression of Eqs. (17) as

$f_{\breve{b}}(u)=\sum_{w \in Z^{n}} f_{\hat{b} \mid \hat{a}}(u \mid w) P[\check{a}=w]$

For the integer LS ambiguity estimator this same result, using a different derivation, was given in Teunissen, (1998b). Equation (21) shows that the PDF of the 'fixed' baseline is equal to an infinite sum of weighted conditional baseline distributions. The conditional distributions $f_{\hat{b} \mid \hat{a}}(u \mid w)$ are shifted versions of one another, while the weights are given by the PMF of $\check{a}$.

In order to visualize the steps involved in constructing $f_{\breve{b}}(u)$, Fig. 3 shows the 1-D case as an example $(n=1, p=1)$. In Fig. 3 the unknown means $a$ and $b$ are taken as zero. Shown are the joint $\operatorname{PDF} f_{\hat{b} . \hat{a}}(u, v)$ of the 'float' solution (top left), the conditional PDF $f_{\hat{b} \mid \hat{a}}(u \mid v)$ (top right), the joint PDF $f_{\breve{b}, \breve{a}}(u, w)$ of the 'fixed' solution (bottom left) and the marginal PDF $f_{\breve{b}}(u)$ of the 'fixed' baseline (bottom right). The conditional $\operatorname{PDF} f_{\hat{b} \mid \hat{a}}(u \mid v)$ is continuous in both $u$ and $v$. When $v$ varies, the conditional PDF moves smoothly, without changing shape, along the line $u=Q_{\hat{b} \hat{a}} Q_{\hat{a}}^{-1} v$ (see Fig. 3, top right). The joint distribution of the 'fixed' solution is located along this same line (see Fig. 3, bottom left). It differs, however, in two ways from $f_{\hat{b} \mid \hat{a}}(u \mid v)$. First, it is not continuous, but discrete along the $w$-axis. Second, since $f_{\breve{b} . \breve{a}}(u, w)=f_{\hat{b} \mid \hat{a}}(u \mid w) P[\check{a}=w]$, it is a downweighted version of the conditional PDF, with the weights $P[\check{a}=w]$ 

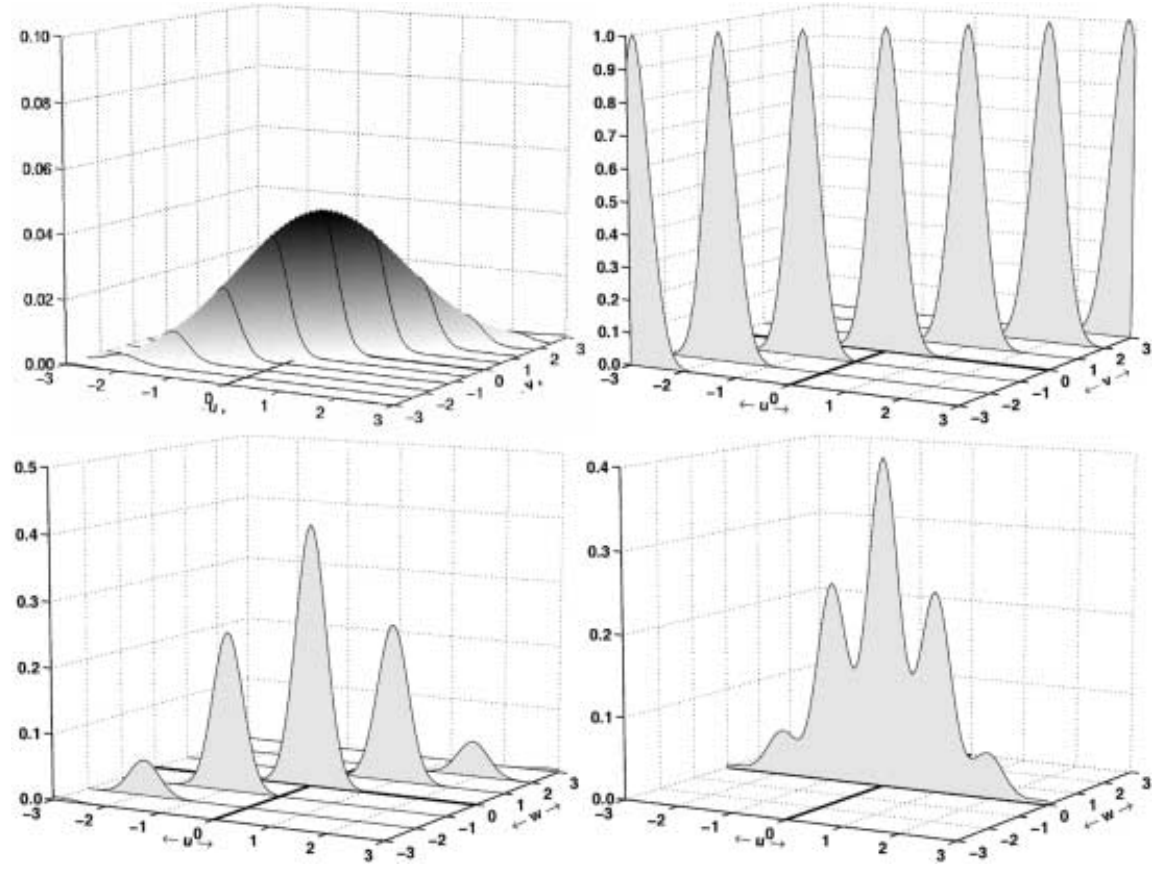

Fig. 3. The construction of $f_{\tilde{b}}(u)$ from $f_{\hat{b}, \hat{a}}(u, v)$ : joint PDF $f_{\hat{b}, \hat{a}}(u, v)$ (top left); conditional PDF $f_{\hat{b} \mid \hat{a}}(u \mid v)$ (top right); joint PDF $f_{\breve{b}, \tilde{a}}(u, w)$ (bottom left); marginal PDF $f_{\tilde{b}}(u)$ (bottom right)

getting smaller the more $w$ differs from zero. The PDF of the 'fixed' baseline is finally obtained from a summation of $f_{\breve{b}, \breve{a}}(u, w)$ along the $w$-axis (see Fig. 3, bottom right). This shows that the PDF of the 'fixed' baseline is symmetric and possibly multimodal. The modes, when present, are equidistantly placed. Whether or not the modes will be present depends on two factors: how fast the PMF $P[\check{a}=w]$ decays as $w$ moves away from the true, but unknown integer ambiguity vector $a \in Z^{n}$, and the distance between the modes. The first factor depends on the precision of the 'float' ambiguities, while the second factor depends on the correlation between the 'float' baseline and the 'float' ambiguities.

With Eq. (21) we are now in a position to give a complete probabilistic evaluation of the 'fixed' baseline. Integration of Eq. (21) over the $b$-centred ellipsoid $E_{b}^{\beta}$ gives

$P\left[\check{b} \in E_{b}^{\beta}\right]=\sum_{w \in Z^{n}}\left\{\int_{E_{b}^{\beta}} f_{\hat{b} \mid \hat{a}}(u \mid w) \mathrm{d} u\right\} P[\check{a}=w]$

or

$P\left[\check{b} \in E_{b}^{\beta}\right]=\sum_{w \in Z^{n}} P\left[\check{b} \in E_{b}^{\beta} \mid \check{a}=w\right] P[\check{a}=w]$

By substituting Eq. (20) we finally obtain

$P\left[\check{b} \in E_{b}^{\beta}\right]=\sum_{w \in Z^{n}} P\left[\chi_{p, \lambda_{w}}^{2} \leq \beta^{2}\right] P[\check{a}=w]$

This result describes the unconditional probability that the 'fixed' baseline estimator resides in the $b$-centred ellipsoid $E_{b}^{\beta}$ of size $\beta$. Equation (22) contains all the ingredients that affect the quality of the 'fixed' baseline estimator. The choice of integer estimator and the quality of the ambiguities are contained in the PMF
$P[\check{a}=w]=\int_{S_{w}} f_{\hat{a}}(x) \mathrm{d} x$, while the contribution to the probability of the baseline of fixing the ambiguities to $w$ is contained in $P\left[\chi_{p, \lambda_{w}}^{2} \leq \beta^{2}\right]$.

\section{Appendix}

\section{Proof of Theorem 1}

Let $f_{\hat{a} \mid \check{a}}(x \mid z)$ be the conditional distribution of $\hat{a}$ given $\check{a}=z$. Then

$P[\hat{a} \in \Omega \mid \check{a}=z]=\int_{\Omega} f_{\hat{a} \mid \check{a}}(x \mid z) \mathrm{d} x$

with $\Omega \subset R^{n}$. This conditional probability can be worked out as

$P[\hat{a} \in \Omega \mid \check{a}=z]=P\left[\hat{a} \in \Omega \mid \hat{a} \in S_{z}\right]=\frac{P\left[\hat{a} \in \Omega, \hat{a} \in S_{z}\right]}{P\left[\hat{a} \in S_{z}\right]}$

The first equality follows from the equivalence of $\check{a}=z$ and $\hat{a} \in S_{z}$, while the second follows from the definition of a conditional probability. Since $P\left[\hat{a} \in \Omega, \hat{a} \in S_{z}\right]$ $=\int_{\Omega \cap S_{z}} f_{\hat{a}}(x) \mathrm{d} x$, it follows that

$P[\hat{a} \in \Omega \mid \check{a}=z]=\int_{\Omega} \frac{f_{\hat{a}}(x) s_{z}(x)}{P\left[\hat{a} \in S_{z}\right]} \mathrm{d} x$

But since $\Omega$ is arbitrary, it follows from Eqs. (A1) and (A2) that $f_{\hat{a}, \breve{a}}(x, z)=f_{\hat{a} \mid \check{a}}(x \mid z) P[\check{a}=z]=f_{\hat{a}}(x) s_{z}(x)$.

\section{Proof of Theorem 2}

We will only prove

$f_{\hat{b}, \hat{a}, \breve{a}}(u, v, w)=f_{\hat{b} \mid \hat{a}}(u \mid v) f_{\hat{a}, \breve{a}}(v, w)$ 
The proof of $f_{\breve{b}, \hat{a}, \breve{a}}(u, v, w)=f_{\hat{b} \mid \hat{a}}(u \mid w) f_{\hat{a}, \breve{a}}(v, w)$ goes along similar lines. Use will be made of the following transformation law for probability density functions. Let $r=T s+t$, in which $r$ and $s$ are random vectors, $T$ and $t$ are known, and matrix $T$ is invertible. Then $f_{r}(r)=\left|\operatorname{det} T^{-1}\right| f_{s}\left(T^{-1}(r-t)\right)$.

In order to prove Eq. (A3), we consider the transformation

$$
\begin{aligned}
& \underbrace{\left[\begin{array}{c}
\hat{b} \\
\hat{a} \\
\check{a}
\end{array}\right]}_{r}= \underbrace{\left[\begin{array}{ccc}
I_{p} & Q_{\hat{b} \hat{a}} Q_{\hat{a}}^{-1} & 0 \\
0 & I_{n} & 0 \\
0 & 0 & I_{n}
\end{array}\right]}_{T} \underbrace{\left[\begin{array}{c}
\hat{b}-Q_{\hat{b} \hat{a}} Q_{\hat{a}}^{-1}(\hat{a}-\alpha) \\
\hat{a}-\alpha \\
\check{a}
\end{array}\right]}_{s} \\
&+\left[\begin{array}{c}
0 \\
\alpha \\
0
\end{array}\right]
\end{aligned}
$$

We first need to determine the PDF of $s$. Since $\hat{a}$ is independent of $\hat{b}-Q_{\hat{b} \hat{a}} Q_{\hat{a}}^{-1}(\hat{a}-\alpha)$, this also holds true for $\check{a}$. We therefore have $f_{s}(x, y, z)=f_{\hat{b} \mid \hat{a}}(x \mid \alpha) f_{\hat{a}-\alpha, \breve{a}}(y, z)$. Substitution of $f_{\hat{a}-\alpha, \breve{a}}(y, z)=f_{\hat{a}, \breve{a}}(y+\alpha, z)$ gives

$f_{s}(x, y, z)=f_{\hat{b} \mid \hat{a}}(x \mid \alpha) f_{\hat{a}, \breve{a}}(y+\alpha, z)$

We can now make use of the transformation law for probability density functions. Matrix $T$ is invertible and its determinant equals 1 . We therefore have $f_{r}(u, v, w)=f_{s}\left(u-Q_{\hat{b} \hat{a}} Q_{\hat{a}}^{-1}(v-\alpha), v-\alpha, w\right)$. This, combined with Eq. (A5) gives

$f_{\hat{b}, \hat{a}, \breve{a}}(u, v, w)=f_{\hat{b} \mid \hat{a}}\left(u-Q_{\hat{b} \hat{a}} Q_{\hat{a}}^{-1}(v-\alpha) \mid \alpha\right) f_{\hat{a}, \breve{a}}(v, w)$ which is identical to Eq. (A3), since an evaluation of the arguments of the conditional baseline density function shows that $f_{\hat{b} \mid \hat{a}}(u \mid v)=f_{\hat{b} \mid \hat{a}}\left(u-Q_{\hat{b} \hat{a}} Q_{\hat{a}}^{-1}(v-\alpha) \mid \alpha\right)$.

\section{References}

Hofmann-Wellenhof B, Lichtenegger H, Collins J (1997) Global positioning system: theory and practice, 4th edn. Springer, Berlin Heidelberg New York

Jonkman NF (1998) Integer ambiguity estimation without the receiver-satellite geometry. LGR Series, no. 18, Delft Geodetic Computing Centre, Delft

Leick A (1995) GPS Satellite Surveying, 2nd edn. John Wiley, New York

Parkinson B, Spilker JJ (eds) (1996) GPS: theory and applications, vols 1 and 2. AIAA, Washington, DC

Strang G, Borre K (1997) Linear algebra, geodesy, and GPS. Wellesley-Cambridge Press

Teunissen PJG (1993) Least-squares estimation of the integer GPS ambiguities. Invited Lecture, Section IV Theory and Methodology, IAG General Meeting, Beijing, August. Also in LGR Series, no. 6, pp 59-74, Delft Geodetic Computing Centre, Delft

Teunissen PJG (1998a) On the integer normal distribution of the GPS ambiguities. Artific Sat 33(2): 49-64

Teunissen PJG (1998b) The distribution of the GPS baseline in case of integer least-squares ambiguity estimation. Artific Sat 33(2): 65-75

Teunissen PJG (1999) An optimality property of the integer least-squares estimator. J Geod 73: 587-593

Teunissen PJG (2001) Integer estimation in the presence of biases. J Geod 75: 399-407

Teunissen PJG, Kleusberg A (eds) (1998) GPS for Geodesy, 2nd edn. Springer, Berlin Heidelberg New York

Teunissen PJG, Joosten P, Tiberius CCJM (2000) Bias robustness of GPS ambiguity resolution. Proc ION GPS-2000, pp 104-112 